\title{
PROPAGACIÓN POR SEMILLAS Y CULTIVO EN MACETA DE Valeriana carnosa SM. (VALERIANOIDEAE, CAPRIFOLIACEAE) EN SAN CARLOS DE BARILOCHE. RÍO NEGRO
}

\author{
SÁNCHEZ, G. ${ }^{1} \&$ RIAT, M. ${ }^{1}$
}

\begin{abstract}
RESUMEN
Valeriana carnosa es una de las especies nativas de la Patagonia más reconocida por la farmacopea local. Sus raíces contienen compuestos químicos utilizados como sedante y son colectadas con un criterio extractivo. El objetivo del trabajo fue obtener información, sobre la propagación por semillas y el cultivo en contenedor, que permitiría establecer futuras producciones disminuyendo el impacto sobre las poblaciones silvestres. Se recolectaron semillas en el Cerro Otto, ciudad de Bariloche en la Provincia de Rio Negro. El ensayo se conformó por un testigo (TO) y 3 tratamientos pregerminativos: Estratificación/ fría-húmeda-30 días (T1), Estratificación/fría-húmeda-10 días (T2), Remojo KNO3-24hs (T3). La siembra se realizó en almácigo y a los 90 días los resultados fueron: TO: 20\%, T1: $62 \%$, T2: $35 \%$, T3: $21 \%$. El repique se hizo en bandeja multiceldas de 25 cavidades y la supervivencia a los 90 días fue del $60 \%$. Los plantines se trasplantaron a maceta soplada ${ }^{\circ} 14$, utilizando 2 sustratos: S1 (Sustrato-Dynamics3) y S2 (sustrato-Suelo/pinocha/ceniza volcánica: 1-1-1). Los valores promedio fueron para altura en $\mathrm{S} 1(12.67 \mathrm{~cm})$ y $\mathrm{S} 2(8,33 \mathrm{~cm})$ y para cantidad de brotes en S1 $(2,73)$ y S2 $(1,67)$. Los resultados son promisorios en cuanto a la reproducción y cultivo en contenedor.
\end{abstract}

Palabras clave: Valeriana, sedante, tratamientos pregerminativos, cultivo en contenedor.

1.- Universidad Nacional De Rio Negro, Instituto de Investigaciones en Agroecología, Recursos Naturales y Desarrollo Rural. Mitre630 S.C. de Bariloche. Argentina. Email: gosanchez@unrn.edu.ar Manuscrito recibido el 14 de abril de 2020 y aceptado para su publicación el 7 deagosto de 2020.

Sánchez G, Riat M. Propagación por semillas y cultivo en maceta de Valeriana carnosa Sm. (Valerianoideae, Caprifoliaceae) en San Carlos de Bariloche. Río Negro FAVE - Ciencias Agrarias 20 (1): 205-218. CC BY-NC-SA 4.0 


\begin{abstract}
Propagation by seeds and container cultivation of Valeriana carnosa Sm. (Valerianoideae, Caprifoliaceae) in San Carlos de Bariloche. Río Negro

Valeriana carnosa is one of the native species of Patagonia most recognized by the local pharmacopoeia. Its roots contain chemical compounds used as a sedative and are collected with an extractive criterion. The objective of the work was to obtain information on seed propagation and container cultivation, which would allow establishing future productions, reducing the impact on wild populations. Seeds were collected in Cerro Otto, in Bariloche, Rio Negro. Control (TO) and 3 pregerminative treatments were performed: Stratification / cold-wet-30 days (T1), Stratification / cold-wet-10 days (T2), Soak KNO3-24 hs (T3). The sowing was done in seedlings and after 90 days the results were: TO: $20 \%, \mathrm{~T} 1: 62 \%, \mathrm{~T} 2: 35 \%, \mathrm{~T} 3: 21 \%$. The pealing was done on a multi-cell tray- 25 and the survival at 90 days was $60 \%$. The seedlings were repackaged to pot $\mathrm{n}^{\circ} 14$, using 2 substrates: S1 (Substrate-Dynamics3) and S2 (substrate-Soil / pine needles / volcanic ash: 1-1-1). The average values were for height in $\mathrm{S} 1(12.67 \mathrm{~cm})$ and $\mathrm{S} 2(8.33 \mathrm{~cm})$ and for the number of outbreaks in S1 (2.73) and S2 (1.67). The results are promising in terms of reproduction and container cultivation.
\end{abstract}

Key words: Valeriana, sedative, pregerminative treatments, container cultivation.

\section{INTRODUCCIÓN}

El género Valeriana L. incluye cerca de 250 especies distribuidas en todo el mundo y en Argentina están citadas 49 especies, 19 de ellas en la región Patagónica (Kutschker, 2011). Valeriana carnosa tiene una distribución amplia, en Argentina incluye la provincia de Neuquén hasta Tierra del Fuego, abarcando las provincias patagónicas en toda su extensión. En Chile se extiende desde la Región de O'Higgins en el norte hasta la Región de Magallanes en el extremo sur, desde la costa pacífica hasta la Cordillera de los Andes. Se desarrolla en laderas suaves y arenosas, bajas y soleadas, en bosques de transición abiertos, en estepa y en alta montaña, entre los 200 y 1300 m.s.n.m. (Zuloaga et al., 2009). V. carnosa Sm. (Fig. 1) es una hierba perenne en roseta con escapos florales de hasta $60 \mathrm{~cm}$ de altura, con uno o más tallos leñosos erectos de hasta $2 \mathrm{~cm}$ de diámetro y raíces con ramificaciones engrosadas. Posee hojas dentadas, carnosas y glabras de color verde azulado claro de entre 6 y $21 \mathrm{~cm}$ de longitud (Correa, 1999).

Es una especie monoica, presenta inflorescencias en panojas terminales o axilares y flores con corola campanulada y textura carnosa. Su fruto es un aquenio con papus plumoso (Fig. 2). Florece en primavera y fructifica a principio del verano (Correa, 1999).

$V$. carnosa más conocida como "ñamkulawen" es una de las cinco especies nativas de la Patagonia Argentina más reconocidas de la farmacopea de los pueblos originarios y sería la especie con propiedades medicinales más popular (Casamiquela, 1999). Históricamente la colecta se realizó des- 
de un punto de vista extractivo (Bandoni, diferentes condiciones de salud y han sido 2003) según la disponibilidad de poblacio- citados como remedios para el hígado, y nes, recurriendo con frecuencia a los mismos vías respiratoria, circulatoria, urinaria y sitios de recolección, generando una presión digestiva, además de tener propiedades sobre las poblaciones naturales que pone en analgésicas, antiinflamatorias, antitumorariesgo su supervivencia en el tiempo. les, antidepresivas y cicatrizantes y estas

Sus raíces y rizomas se usan tradicio- propiedades habrían determinado que la nalmente mediante decocción para tratar planta sea reconocida como "el remedio

Figura 1. Características morfológicas de Valeriana carnosa.

Figure 1. Morphological characteristics of Valeriana carnosa.
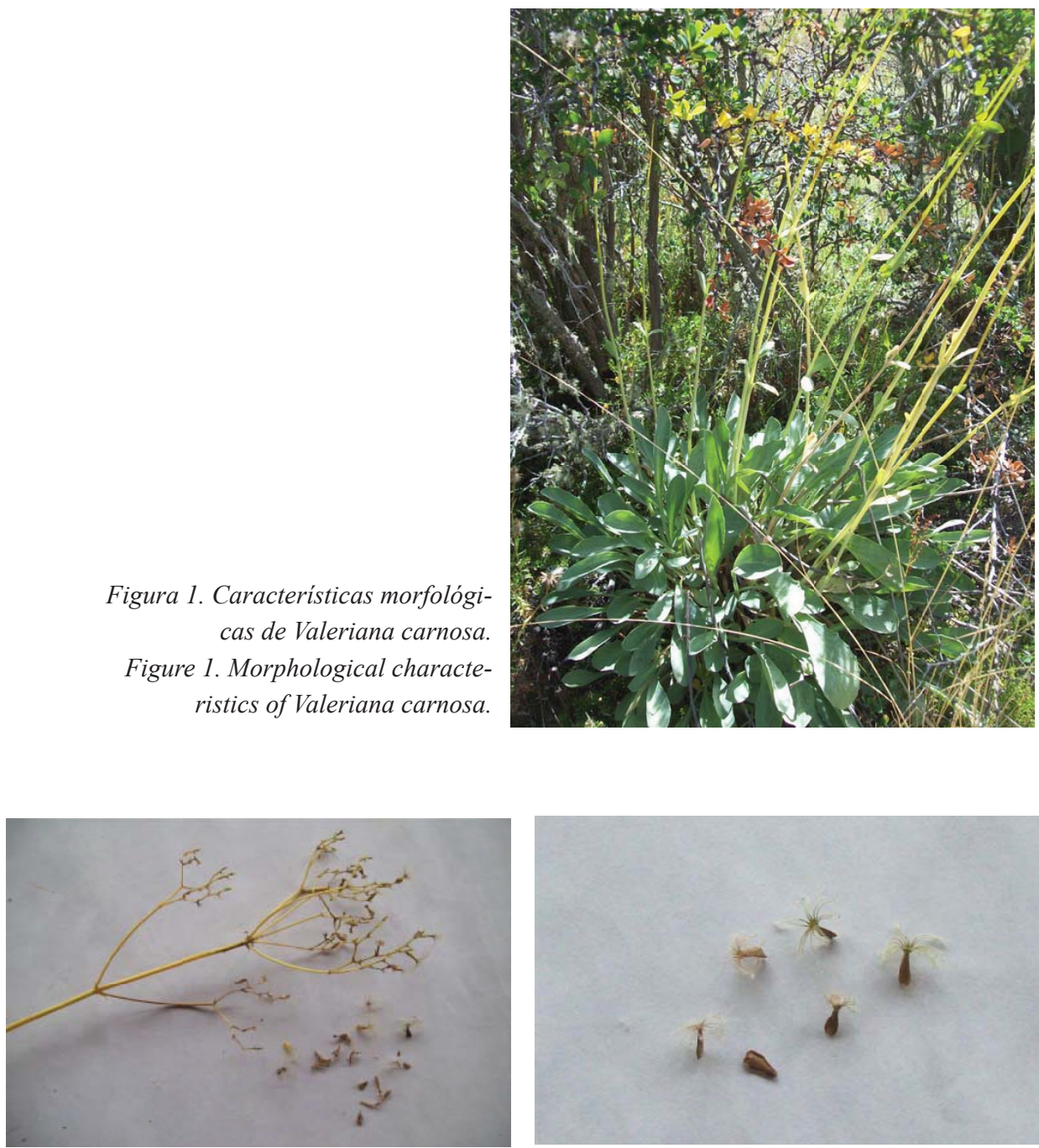

Figuras 2 y 3. Características morfológicas de Valeriana carnosa. Escapo y aquenios plumosos. Figures 2 and 3. Morphological characteristics of Valeriana carnosa. Escape and feathery achenes. 
que cura las siete enfermedades"(Estomba et al., 2005; Morales y Ladio, 2012; Richeri et al., 2013). Los estudios químicos que se están llevando adelante analizan y comparan la variabilidad de los contenidos de los compuestos fenólicos y la actividad antioxidante de los extractos etanólicos presentes (Guajardo et al., 2018).

Las raíces engrosadas de muchas especies del género Valeriana contienen numerosos compuestos químicos que son tradicionalmente utilizados por la industria homeopática (Leathwood et al., 1982; Donath et $a l, .2000)$ y las principales fuentes de obtención de estos compuestos con fines comerciales provienen de la especie $V$. officinalis L. En las últimas décadas tanto la industria homeopática como la cosmética y la farmacéutica comenzaron a emplear extractos naturales de otras especies del género Valeriana como reemplazo de la fuente de materia prima (Bach et al., 2010), sin que existan legislaciones que regulen los procesos extractivos y que promuevan su cultivo. En la Patagonia este proceso pasó de ser extractivo a extractivista (Bandoni, 2003) y con los manejos actuales, la disponibilidad y uso efectivo de las plantas con órganos subterráneos útiles no dejan de ser vulnerables a perderse. Este fenómeno de cambio de uso ha sido relevado en otras comunidades patagónicas y ha sido asociado a factores socioculturales y ecológico-ambientales, existiendo evidencias de una menor abundancia en los ambientes naturales y una mayor dificultad para su búsqueda (Ochoa y Ladio, 2011). Este proceso indicaría la necesidad de propiciar acciones tendientes a una mayor concientización del valor de estos recursos para la región.

Dentro del proyecto específico del INTA "Plataformas tecnológicas y comerciales, para aromáticas cultivadas-nativas y medi- cinales" para Patagonia donde V. Carnosa es una de las especies de interés (INTA, 2013), se están evaluando métodos de multiplicación agámica, estableciendo un sistema de macro propagación y cultivo de bajo costo a partir de enraizamiento de esquejes obtenidos de rizomas de diferentes diámetros y se espera que los resultados obtenidos permitan en el mediano plazo, iniciar un manejo económico productivo racional (Nagahama et al., 2016).

Los antecedentes encontrados de reproducción por vía sexual de Valeriana clarionifolia indican que la germinación registró valores del 57\% al momento de la recolección y que los porcentajes decrecen (pérdida de viabilidad) con el almacenamiento de las semillas. De los diferentes tratamientos pregerminativos ensayados el mayor número de semillas germinadas, de un año de almacenamiento, se obtuvo con estratificación ( $\pm 5^{\circ} \mathrm{C}, 7$ días), logrando después de los 60 días un porcentaje de germinación del 27\% \pm 11 (Berastegui et al., 2016).

Actualmente no hay antecedentes de propagación por vía sexual y cultivo de $V$. carnosa en la Argentina y contar con la información sobre la reproducción a partir de semillas y su cultivo en maceta permitiría el desarrollo y la implantación de futuras producciones a campo y así disminuir la presión de la extracción en poblaciones silvestres.

El objetivo general de este trabajo fue evaluar los métodos de propagación por semillas y la obtención de plantines en contenedor de $V$. carnosa, que permitan generar información para establecer futuros cultivos y producciones. Dentro de los objetivos específicos se planteó evaluar los métodos de colecta, limpieza y calidad de semillas; determinar la influencia de los distintos tratamientos pregerminativos sobre la ruptura 
de la latencia de los aquenios y compararlos según los porcentajes de germinación obtenidos. Además, evaluar la respuesta al crecimiento de los plantines (altura y cantidad de tallos), utilizando dos sustratos de cultivo: uno comercial y otra mezcla con materiales de origen local.

\section{MATERIALES Y MÉTODOS}

Los ensayos se llevaron a cabo en las instalaciones del vivero del Servicio Forestal Andino de la ciudad de San Carlos de Bariloche $\left(41^{\circ} 09^{\prime} \mathrm{S}, 71^{\circ} 18^{\prime} \mathrm{O}\right.$, altitud 893 m) a cargo de la Universidad Nacional de Rio Negro donde se realizan las prácticas de la carrera Tecnicatura en Viveros.

Ensayo 1: Obtención de plantines de V. carnosa a partir de la aplicación de distintos tratamientos pre germinativos.

La colecta del material se realizó a fines de febrero de una población de $V$. carnosa ubicada en la ladera norte del Cerro Otto en la Ciudad de San Carlos de Bariloche (41 ${ }^{\circ}$ $08^{\prime} \mathrm{S}, 71^{\circ} 20^{\prime} \mathrm{O}$, altitud $1150 \mathrm{~m}$ ). La condición fenológica para la colecta fue que los escapos estén secos (Fig. 3). Los frutos se dejaron orear durante 3 días y se guardaron en bolsas de papel hasta el momento de la limpieza, la cual se realizó utilizando distintos tamices (20 mm, $5 \mathrm{~mm}, 2 \mathrm{~mm}$ ) que permitieron separar los aquenios de los restos de las inflorescencias y hacer una selección de los distintos calibres. Como consecuencia de la limpieza se decidió dividir el ensayo en 2 lotes según los calibres seleccionados y sembrarlos por separado, considerando que el tamaño de la semilla está directamente relacionado con la cantidad de reservas disponibles y con la viabilidad (Ju- seau et al., 2013). Se analizaron la pureza y las variables físicas de los dos lotes: peso de 1000 semillas, cantidad de semillas por gramo y calibre según normas de La International Seed Testing Association (ISTA, 2016). Para evaluar la ruptura del letargo de los aquenios se propuso la realización de tratamientos pregerminativos. Considerando los antecedentes encontrados para V. clarionifolia (Berastegui et al., 2016) se establecieron 1 testigo sin tratamiento previo (T0) y 3 tratamientos pregerminativos: Estratificación fría- húmeda- 30 días (T1), Estratificación fría- húmeda- 10 días (T2), Remojo en Nitrato de Potasio- $200 \mathrm{mg} / \mathrm{l}$ durante 24 hs (T3). Se plantearon 3 repeticiones de 200 aquenios por tratamiento, se estratificaron por separado en un recipiente plástico con vermiculita húmeda y se ubicaron en la heladera a una temperatura de $6^{\circ} \mathrm{C} \pm 1$ y se escalona la realización de los tratamientos pregerminativos para hacer coincidir la fecha de siembra.

La siembra se realizó en almácigo en el mes de junio, utilizando un sustrato mezcla compuesto de turba Sphagnum (Origen Tierra del fuego- $\mathrm{pH} 4,2$ ) y perlita en proporciones 2-1 respectivamente. $\mathrm{El} \mathrm{pH}$ de la turba se corrigió a valores neutros con la incorporación de $240 \mathrm{~g}$ de óxido de calcio por bolsa de turba $\left(120 \mathrm{dm}^{3}\right)$. Se midió $\mathrm{pH}$ a los 7 días de la corrección, con una relación 1+5 v/v, (Barbaro et al., 2011), utilizando para ello un phmetro portátil marca Hanna.

Los almácigos se ubicaron en una sala de cultivo con temperaturas entre $18 \mathrm{y}$ $22^{\circ} \mathrm{C}$, en estanterías con iluminación artificial con fotoperiodo de 12 horas luz- día regulado con timmer. El riego se realizó en forma manual según requerimiento de la germinación. 
Considerando el desarrollo de 2 pares de nomofilos como criterio de crecimiento de las plántulas, en el mes de agosto se realizó el repique a bandeja multiceldas de de 25 cavidades de $167 \mathrm{~cm} 3$ utilizando el mismo sustrato empleado para la siembra. Las bandejas se ubicaron dentro de un invernadero sobre mesadas con calefacción basal con temperatura del sustrato entre 18 y $22^{\circ} \mathrm{C}$ controlada con termostato y sistema de riego por microaspersión con una frecuencia de 3 días a la semana durante 20 minutos. Para la etapa de establecimiento del cultivo la fertilización se realizó en forma manual una vez por semana utilizando Hakaphos ${ }^{\circledR}$ violeta $(1 \mathrm{~g} / \mathrm{l})$.

En relación a la germinación se evaluó poder germinativo a los 90 días desde la siembra (PG90) considerando la emergencia de los cotiledones como criterio para determinar semillas germinadas (Rao et al., 2006). Con posterioridad al repique se evaluaron el establecimiento de las plántulas y los porcentajes de supervivencia en bandeja multiceldas para la fecha del trasplante. Se realizó un análisis ANOVA $(p>0,05)$ con test de Tukey para evaluar diferencias significativas entre tratamientos pregerminativos.

La calidad del plantin al momento del trasplante a maceta soplada de $14 \mathrm{~cm}$ de diámetro (M14) se evaluó en relación al desarrollo del sistema radical, considerando que la formación de un cepellón compacto, la calidad, cantidad y ubicación de las raíces darán una idea del control de las variables ambientales, el riego y de la nutrición del cultivo (Styer y Koransky, 2000).

\section{Ensayo 2: Cultivo en maceta de V. carnosa}

El trasplante se realizó en el mes de diciembre a maceta soplada $n^{\circ} 14$ y se utilizaron 2 sustratos: S1 (Sustrato comercial Dynamics $3^{\circledR}$ ) compuesto de turba Sphagnum, perlita y fertilizantes $1,5 \mathrm{~g} / 1$ (N-P-K y microelementos) y S2 (sustrato mezcla local: Suelo, pinocha compostada, ceniza volcánica y aserrín, en proporciones 3-3-31 respectivamente). Los elementos para la formulación del sustrato mezcla (S2) son de origen local, Se consideró el uso de suelo de los alrededores de Bariloche, ceniza volcánica proveniente de la erupción del Volcán Puyehue en junio de 2011, acícula de pino de la especie Pseudotsuga menziesii (pino oregón) presente en plantaciones forestales de la zona y aserrín residual sin compostar proveniente de aserraderos de Bariloche. Se incorporó al sustrato una dosis de $1,5 \mathrm{~g} / 1$ de fertilizante de liberación lenta Osmocote ${ }^{\circledR}$ - 3M (NPK 16-8-12), para compensar los aportes de fertilizantes del sustrato comercial Dynamics $3^{\circledR}$.

Las propiedades físicas y físico-químicas de S1 y S2, fueron analizadas en el Laboratorio de Sustratos y Aguas del Instituto de Floricultura del INTA y los resultados se muestran en la tabla 1 (Barbaro et al., 2014; Ridiero y Mazzoni, 2016).

Las plantas se ubicaron en el exterior sobre membrana geotextil, con un sistema de riego por microaspersión y con una frecuencia de 3 días a la semana durante 30 min en los meses de alta evapotranspiración del cultivo (de diciembre a mayo). La fertilización de todo el ensayo se realizó con fertilizante de liberación lenta Osmocote- 3M (NPK 16-8-12) con una dosis de $1.5 \mathrm{~g} / 1$ por maceta. 
Tabla 1. Propiedades fisicas- químicas del sustrato comercial Dynamics $3^{\circledR}$ y Sustrato mezcla local. Densidad aparente (DAP), espacio poroso total (EPT), porosidad de aire (PA), capacidad de retención de agua (CRA), MO\%, granulometría (\% por fracción de tamaño), pH y conductividad eléctrica (CE).

Table 1. Physical-chemical properties of the commercial substrate Dynamics $3^{\circledR}$ and Substrate local mix. Apparent density (DAP), total porous space (EPT), air porosity (PA), water retention capacity (CRA), $M O \%$, particle size (\% by size fraction), $\mathrm{pH}$ and electrical conductivity (CE).

\begin{tabular}{|c|c|c|c|}
\hline & Unidad & $\begin{array}{c}\text { Dynamics } 3^{(\otimes)} \\
\text { S1 }\end{array}$ & $\begin{array}{l}\text { Sustrato Mezcla Local } \\
\text { S2 }\end{array}$ \\
\hline DAP & $\mathrm{Ton} / \mathrm{m}^{3}$ & 0,08 & 0.559 \\
\hline $\mathrm{pH}$ & & 5.11 & 6.2 \\
\hline CE & $\mathrm{dS} / \mathrm{m}$ & 0.91 & 0.17 \\
\hline MO & $\% \mathrm{~m} / \mathrm{m}$ & $s / d$ & $12 \%$ \\
\hline EPT \% & $\%$ & 95 & 75 \\
\hline PA $\%$ & $\%$ & 41 & 22 \\
\hline CRA \% & $\%$ & 54 & 53 \\
\hline \multirow{4}{*}{ Granulometría } & $>3.35 \mathrm{~mm}$ & 33 & 0 \\
\hline & $3.35-1.0 \mathrm{~mm}$ & 27 & 7 \\
\hline & $<1.0 \mathrm{~mm}$ & 39 & 93 \\
\hline & & $\begin{array}{c}\text { Barbaro et al., } \\
2014\end{array}$ & Ridiero, 2016 \\
\hline
\end{tabular}

Para la realización del ensayo se plantearon 3 repeticiones de 5 plantas por sustrato utilizado con un diseño en bloques aleatorizados y se evaluaron los porcentajes de supervivencia, la altura de planta $(\mathrm{cm})$ y la cantidad de tallos por planta, en la fecha del trasplante y al final de la primera temporada de crecimiento en el mes de abril. Se realizó un análisis ANOVA $(\mathrm{p}>0,05)$ con test de Tukey para evaluar las variables de crecimiento de las plantas en relación a los sustratos utilizados.

\section{RESULTADOS}

Ensayo 1: Obtención de plantines de V. carnosa a partir de la aplicación de distintos tratamientos pre germinativos.

La limpieza con tamices permitió separar los aquenios y dividirlos en 2 lotes según el calibre: calibre 1 ( $\geq 5 \mathrm{~mm}$ ) y calibre 2 $(\leq 5 \mathrm{~mm})$. Los porcentajes de pureza fueron para el calibre 1: 79\% y para el calibre 2: $67 \%$, mientras que el peso de 1000 semillas fueron para el calibre 1: $2,92 \mathrm{~g}$ y para el calibre $2: 1,54 \mathrm{~g}$. 
Para los aquenios del lote calibre 2 ( $\leq$ $5 \mathrm{~mm}$ ) no se obtuvieron porcentajes de germinación en ninguno de los tratamientos realizados. Con respecto al poder germinativo a los 90 días (PG90) los porcentajes de germinación para el lote calibre 1 ( $\geq$ $5 \mathrm{~mm}$ ) fueron para el (T0) un 20\%, para el T1 (EFH-30) un 60\%, para el T2 (EFH-10) un $35 \%$ y para el (T3) un $21 \%$. Como se observa en la Figura 4 los porcentajes de germinación obtenidos para el T1 (EFH-30) presentan diferencias significativas con respecto al testigo y a los otros tratamientos, aumentando el poder germinativo del lote al realizar estratificación fría y húmeda.

El porcentaje de supervivencia entre todos los tratamientos luego del repique fue del $60 \%$ y las plántulas que se establecieron tuvieron una buena respuesta al cultivo en bandeja multiceldas (Figura 5). En cuanto a la calidad de plantín, como se observa en la Figura 6, el sistema radical formó un cepellón firme y un desarrollo de raíces homogéneo en todo el volumen de la celda.

\section{Ensayo 2: Cultivo en maceta de \\ V. carnosa}

El porcentaje de supervivencia al reenvasado a maceta $n^{\circ} 14$ luego de la primera temporada de crecimiento fue del $100 \%$ para el sustrato S1 (Dynamics $3^{\circledR}$ ) y del $80 \%$ para el sustrato S2 (Suelo/ pinocha/ ceniza volcánica/ aserrín: 3-3-3-1). En relación a la altura de plantas los resultados presentaron diferencias significativas entre los sustratos utilizados. Para el sustrato S1 la altura promedio de las plantas fue de $12,27 \mathrm{~cm}$ mientras que para el sustrato $\mathrm{S} 2$ fue de $8,33 \mathrm{~cm}$ (Figura 7). En cuanto a la cantidad de tallos los resultados no difieren significativamente entre sí en relación a los sustratos utilizados. Para el sustrato S1, el promedio fue de 2,73 tallos, mientras que para el sustrato S2 el promedio fue de 1,67 tallos (Figura 8).

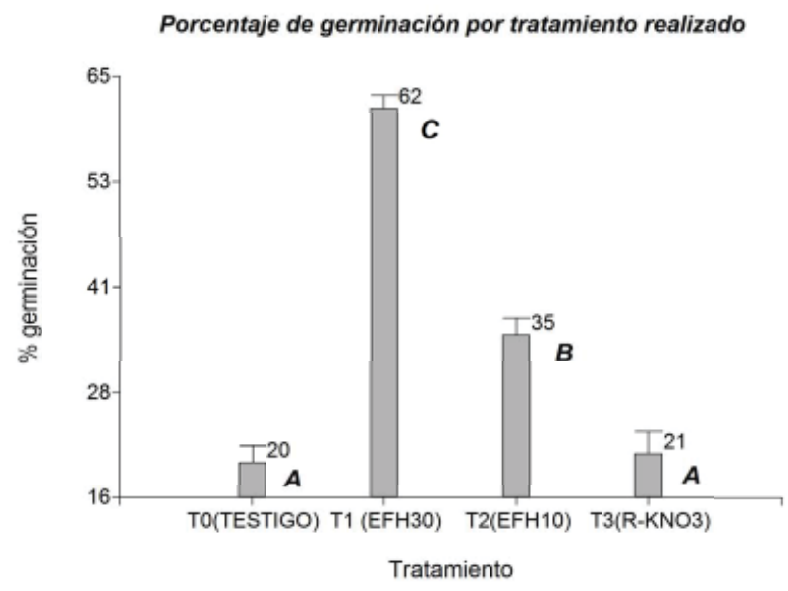

Figura 4. Porcentajes de germinación de Valeriana carnosa a los 90 días (PG90) por tratamientos pregerminativos realizados en aquenios calibre $1(\geq 5 \mathrm{~mm})$ Letras diferentes indican diferencias significativas. Media + - error estándar (4,33; Test de Tukey para $p>0,05)$.

Figure 4. Germination percentages of Valeriana carnosa at 90 days (PG90) by pregerminative treatments performed on achenes caliber $1(\geq 5 \mathrm{~mm})$. 


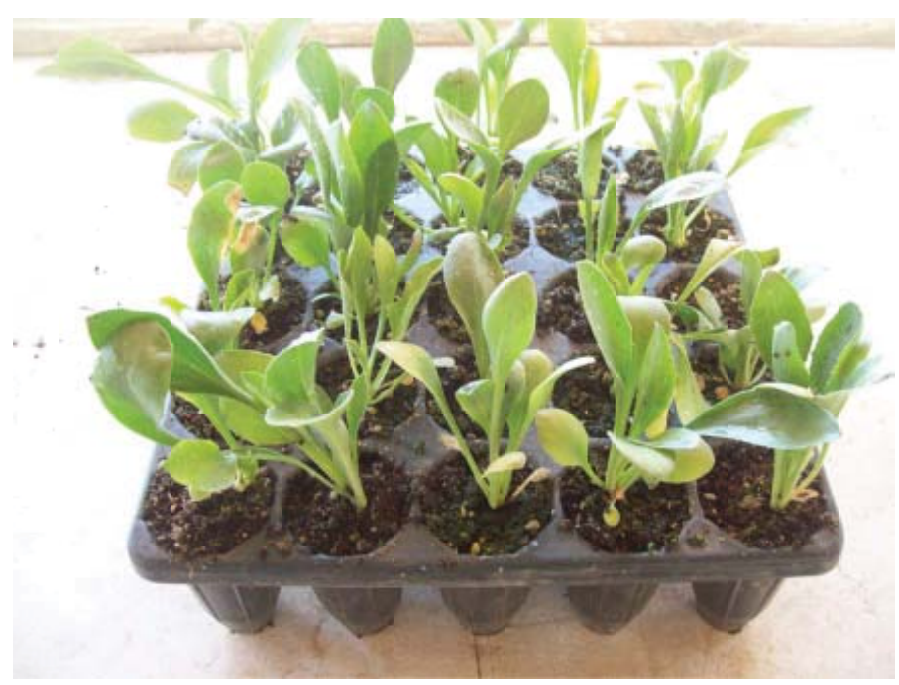

Figura 5. Cultivo en bandeja multiceldas 25 de Valeriana carnosa. Figure 5. Culture in a multi- cell tray 25 of Valeriana carnosa.

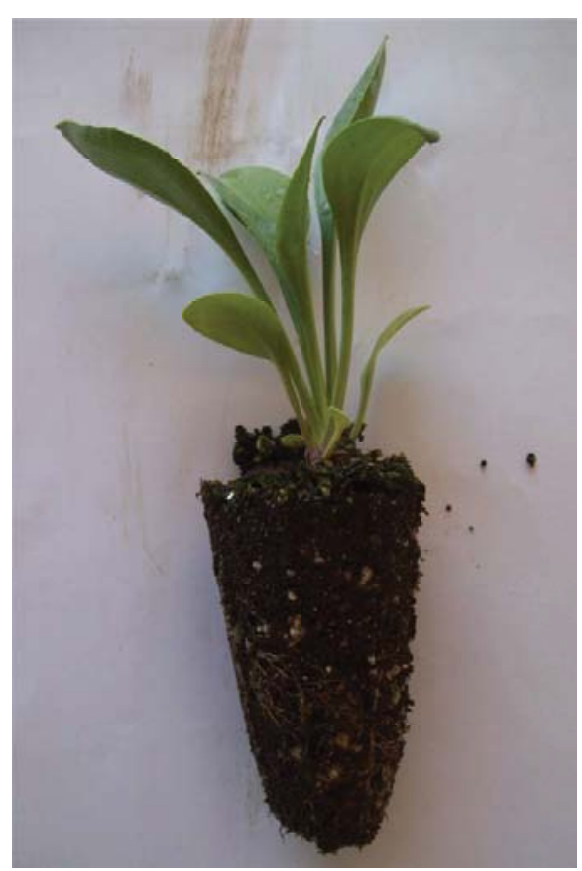

Figura 6. Plantin de Valeriana carnosa. Figure 6. Seedlings of Valeriana carnosa. 
G. Sánchez et al.

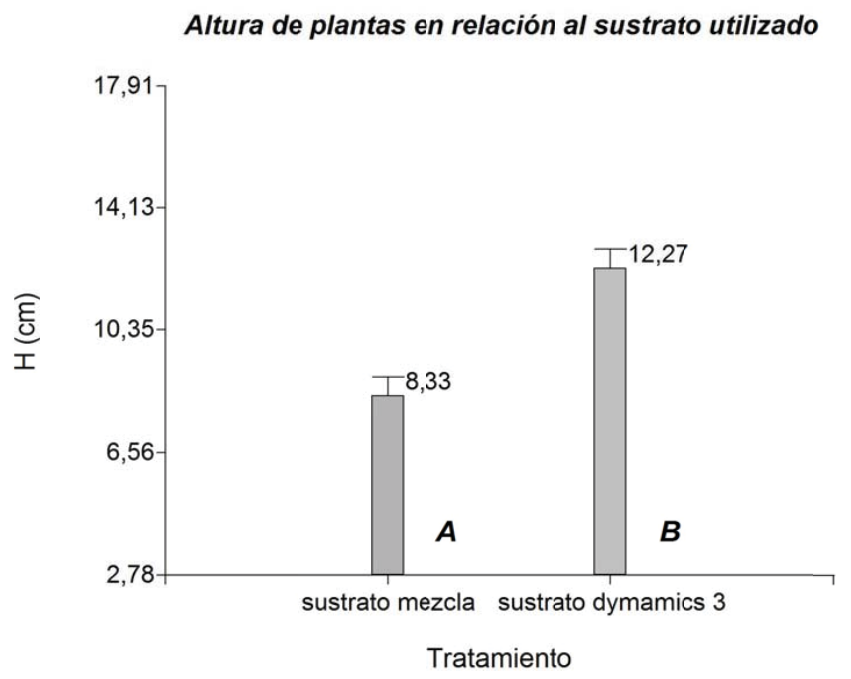

Figura 7. Altura de plantas de Valeriana carnosa en relación al sustrato utilizado. Letras diferentes indican diferencias significativas. Media + - error estándar (5,15; Test de Tukey para $p>0,05)$

Figure 7. Height of Valeriana carnosa plants in relation to the substrate used.

\section{Cantidad de tallos en relación al sustrato utilizado}

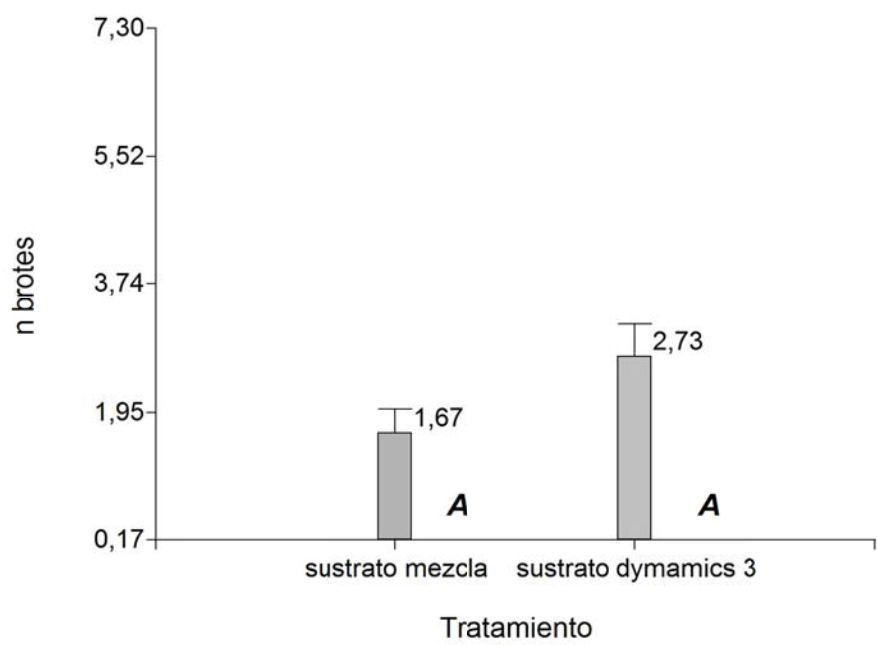

Figura 8. Cantidad de tallos de Valeriana carnosa en relación al sustrato utilizado. Letras diferentes indican diferencias significativas. Media + - error estándar (2,36; Test de Tukey para $p>0,05)$.

Figure 8. Number of Valeriana carnosa stem in relation to the susbstrate used. 
Como se observa en la Figura 9, el desarrollo radicular en el sustrato Dynamics $3^{\circledR}$ (S1) fue más homogéneo colonizando todo el volumen de sustrato. En el sustrato mezcla (S2) en la que se observa el desarrollo del sistema radicular en la periferia del sustrato sin colonizar la totalidad de su volumen.

\section{DISCUSIÓN}

Ensayo 1: Obtención de plantines de V. carnosa a partir de la aplicación de distintos tratamientos pre germinativos.

Al dividir el ensayo en 2 lotes según los calibres seleccionados después de la limpieza y decidir sembrarlos por separado, considerando lo que dice Juseau (2013) que el tamaño de la semilla está directamente relacionado con la cantidad de reservas disponibles y con la viabilidad, permitió ajustar la evaluación del ensayo en relación al poder germinativo, ya que como hace referencia Escobar (2008) el calibre de la semilla puede afectar significativamente el comportamiento de otras variables que la califican así como también su comportamiento durante la etapa de siembra. Esta clasificación previa permitió mejorar la respuesta del lote 2 en relación al Poder germinativo. Si ambos calibres hubieran sido manejados como un solo lote los resultados en el variable poder germinativo hubieran sido menores porque dentro del lote se incluyen muchas semillas no viables. Esto refuerza y valida la necesidad de conocer las características específicas de las semillas para definir patrones de selección y limpieza que mejoren la calidad de los lotes de semillas (ISTA, 2016).

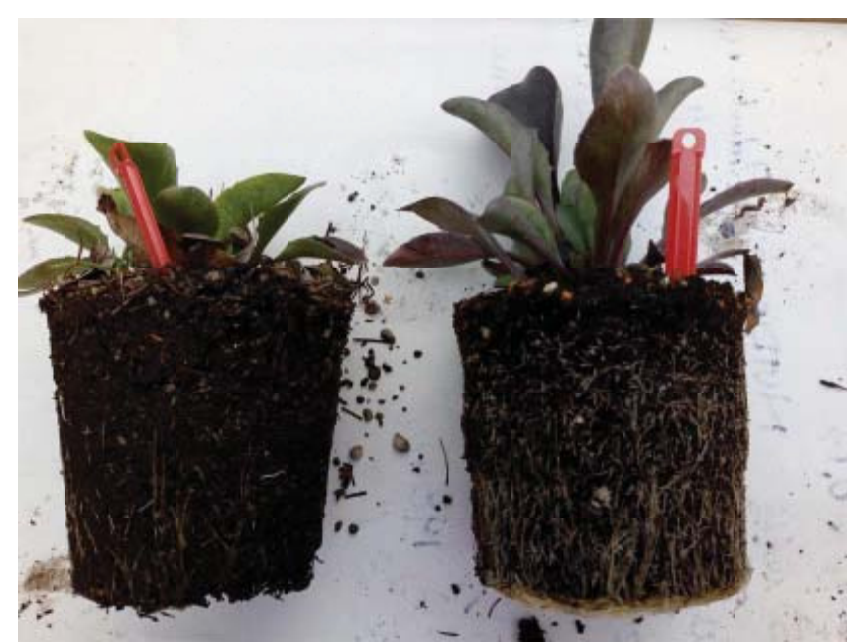

Figura 9. Desarrollo radicular de Valeriana carnosa en relación al sustrato utilizado. Izquierda sustrato sustrato mezcla (S2) y derecha Dynamics 3 (S1).

Figure 9. Root development of Valeriana carnosa in relation to the susbstrate used. Left right mix substrate (S2) and Dynamics 3 substrate (S1) 
El desarrollo del cultivo en bandeja multiceldas dentro del invernadero, el manejo de las variables ambientales como la temperatura, la frecuencia y la duración del riego y la fertilización posibilitó la formación de un cepellón compacto y un desarrollo homogéneo de las raíces en todo el volumen de la celda. Estas características, que según Styer y Koransky (2000) son indicadores de calidad, permitieron realizar el trasplante a maceta a los 4 meses desde la fecha del repique.

\section{Ensayo 2: Cultivo en maceta de \\ V. carnosa}

Los resultados obtenidos en la calidad del cepellón y distribución de raíces expresan una relación directa con la calidad de los sustratos utilizados. Una mejor distribución y calidad de raíces observada en el tratamiento con el sustrato S1, en relación a las plantas cultivadas en el sustrato S2, podrían estar relacionadas con la cantidad y calidad del espacio poroso de los sustratos. S1 presenta un 95\% de EPT en contraposición al $75 \%$ de S2. La calidad de este espacio poroso definida por la distribución entre agua (CRA\%) y aire (PA\%) muestra una mayor retención de agua y menor disponibilidad de aire para el sistema radicular en S2. Si bien los valores de CRA\% resultan iguales representan en S1 una proporción mucho mayor del espacio poroso $(70 \%$ en $\mathrm{S} 1$ y $56 \%$ en S2). Esto se podría explicar analizando la distribución de las fracciones granulométricas, en S2 la fracción menor de $1 \mathrm{~mm}$ alcanza un $93 \%$, definiendo poros de menor tamaño y por lo tanto mayor succión mátrica lo que reduce la disponibilidad de agua. Paralelamente, el S2 presenta la mitad del espacio poroso de aire respecto del S1. Estudios posteriores a la erupción, analizaron el uso en sustratos de fracciones granulométricas menores a $1 \mathrm{~mm}$ de cenizas volcánicas del complejo Puyehue-Cordón Caulle, se observando un aumento marcado de la retención de agua en tensiones de 20 a $100 \mathrm{hP}$ (Riat et al., 2013), por lo que el tamaño de las partículas de las cenizas es una variable importante a la hora de decidir su uso en la formulación de sustratos (Barbaro et al.,2015) y esto según los resultados obtenidos, resulta importante para la mejora de la calidad de las plantas de Valeriana carnosa.

\section{CONCLUSIONES}

Se concluye que con la aplicación de tratamientos pregerminativos se logra romper el letargo de los frutos. Con la estratificación fría- húmeda durante 30 días (T1) se obtiene un mayor poder germinativo en relación a los otros tratamientos realizados y al testigo. Después de la primera temporada de crecimiento los porcentajes de supervivencia en maceta permiten concluir que la especie tiene una buena adaptación al cultivo. Se concluye que es posible el cultivo en maceta de la especie, obteniendo plantas con una buena adaptación al riego por microaspersión y a la fertilización en el primer año desde la siembra. Comparando la respuesta en la calidad de las plantas con los 2 sustratos utilizados, resulta importante la calidad de los mismos, tanto en el EPT \% cómo en la PA\%. Los resultados son promisorios en cuanto a la reproducción y cultivo en maceta de Valeriana carnosa y permitirían avanzar en la evaluación de otras variables como el crecimiento de rizomas y el peso seco del desarrollo radical, para generar información que permita desarrollar en el futuro protocolos de cultivo en viveros. 


\section{BIBLIOGRAFÍA}

Bach HG, Gurni AA, Fortunato RH, Wagner M. 2010. Estudio Anatómico y Fitoquímico de "Valerianas" comercializadas en la Patagonia Argentina. X Simposio Argentino, XIII Simposio Latinoamericano de Farmacobotánica. Córdoba, Argentina.

Bandoni AL. 2003. Los recursos aromáticos en Latinoamérica. $2^{\mathrm{a}}$ edición. Buenos Aires. 158 pp.

Barbaro LA, Karlanian MA, Imhoff L, Morisigue DE. 2011. Caracterización de la turba subtropical del departamento Islas del Ibicuy (Entre Ríos, Argentina). Agriscientia 28(2): 137-145.

Barbaro LA, Imhoff L, Morisigue DE. 2014. Evaluación de sustratos formulados con corteza de pino, pinocha y turba subtropical. Cienc Suelo (Argentina) 32(2): 149-158, 2014.

Barbaro LA, Healy VI, Karlanián MA \& Mazzoni A. 2015. Ceniza Volcánica como alternativa a la perlita en la formulación de sustratos para plantines florales. Cienc Suelo (Argentina) 33(2): 213-219, 2015

Berastegui AM, Gratt A, Fernández S, Ricci M, Belen R. 2016. Valeriana clarionifolia (Valerianáceas). Estrategia de cultivo in vitro. Dominguezia 32(2):60. Noviembre de 2016. Ciudad Autónoma de Buenos Aires - República Argentina. Versión digital. ISSN 16696859

Casamiquela RM. 1999. Proyecto etnobotánico de la Patagonia: Primer Informe. en E. Suárez, R. H. Fortunato, M. Elechosa, R. Casamiquela, E. Saavedra y B. N. Timmermann (eds.). Aspectos Técnicos, Culturales, Políticos y Legales de la bioprospección en Argentina. Buenos Aires: ICBG. INTA. CENPAT. UNP y UA.

Correa MN. 1999. Flora Patagónica. Tomo 8. Parte VI. Colección Científica del INTA. 536 pp.
Donath F., Quispe S., Diefenbach K., Maurer A., Fietze I., Roots I. 2000. Critical evaluation of the effect of valerian extract on sleep structure and sleep quality. Pharmacopsychiatry. 2000 Mar; 33(2):47-53

Escobar R. 2008. Manejo de semillas forestales. Quillón - Chile

Estomba D, Ladio A, Lozada M. 2005. Plantas medicinales utilizadas por una comunidad Mapuche en las cercanías de Junín de los Andes, Neuquén. Boletín Latinoamericano y del Caribe de Plantas Medicinales y Aromáticas 4 (6): 107-112.

Gómez Paternina DA, Barros Algarra R, Sierra Sarmiento M. 2018. Manual del Buen Uso de Plantas Medicinales. Fundación Universitaria Agraria de Colombia-UNIAGRARIA. $44 \mathrm{p}$.

Guajardo J, Gastaldi B, González S, Nagahama N. 2018. Variabilidad de compuestos fenólicos en Variabilidad de compuestos fenólicos en diferentes estadios fenológicos en dos poblaciones de Valeriana carnosa Sm. (Valerianoideae, Caprifoliaceae) en Patagonia. Boletín Latinoamericano y del Caribe de Plantas Medicinales y Aromáticas 17 (4): 381 - 393. Joseau JM, Conles MY y Verzino GE. 2013. El cultivo de plantas leñosas en viveros y a campo. Colección: Conservación de recursos forestales nativos de Argentina. $1^{\text {a }}$ ed. Editorial Brujas. Córdoba. 311 pág.

Kutschker A. 2011. Revisión del Género Valeriana (Valerianaceae) en Sudamérica austral. En Gayana Bot. 68(2): 244-296. Concepción, Chile. 2011

Leathwood PD, Chauffard F, Heck E, Muñoz BR. 1982. Aqueous extract of valerian root (Valeriana officinalis L.) improves sleep quality in man. Pharmacol Biochem Behav. 1982 Jul; 17(1):65-71. 
Molares S y Ladio AH. 2012. Plantas aromáticas con órganos subterráneos de importancia cultural en la Patagonia Argentina: una aproximación a sus usos desde la etnobotánica, la percepción sensorial y la anatomía. Darwiniana 50(1): 7- 24.

Nagahama N, Bach HG, Opazo WJ, Miserendino E, Arizio CM, Manifesto MM, Fortunato RH. 2016. Aprovechamiento sustentable de recursos genéticos nativos: Valeriana carnosa Sm., estudio de caso de una planta medicinal Patagónica. Dominguezia 32(2):69.

Ochoa JJ, Ladio A. 2011. Pasado y presente del uso de plantas silvestres con órganos de almacenamiento subterráneos comestibles en la patagonia. Bonplandia 20(2): 265-284.

Orell R. 2013. INTA. Proyecto Nacional Específico: Plataformas tecnológicas y comerciales para plantas aromáticas cultivadas nativas y medicinales. Código de Planificación PNHFA 1106093.

Rao NK, Hanson J, Dulloo ME, Ghosh K, Nowell D and Larinde M. 2006. Manual of seed handling in genebanks. Handbooks for Genebanks No. 8. Bioversity International, Rome, Italy.

Riat M, Laos F, Alzogaray S, Arrigoni JP, Paladino G y Naón S. 2013. Efectos de la ceniza volcánica del complejo Puyehue-Cordón Caulle en la formulación de sustratos y la absorción de nutrientes. Libro de resúmenes del XXXVI Congreso Argentino de Horticultura (ASAHO). Tucumán. En Horticultura Argentina 32(79): 133. Sep.-dic. 2013. ISSN 1851-9342.
Ridiero E, Mazzoni A. 2016. Evaluación de sustratos en el cultivo de Petunia x hybrida bajo condiciones de invernadero en Bariloche. Comunicación técnica №255.Área Desarrollo Rural. Ediciones INTA. ISSN 1667-4006.

Styer RC, Koransky D. 2000. Controlling the root to shot radio. En Growers Talks on Plugs Plugs 3. Chapter 3. Edited by Vender Velde Jaine. Ed Ball Publishing 2000. 106 pág.

The International Seed Testing Association (ISTA). 2016. Reglas Internacionales para el Análisis de las Semillas. Zürichstr. 50, CH8303 Bassersdorf, Suiza (C2016 International Seed Testing Association (ISTA). Online ISSN 2310-3655.

Zuloaga FO, Morrone O, Belgrano M. 2009. Catálogo de las Plantas Vasculares del Cono sur. Instituto de Botánica Darwinion. IBODA-CONICET. http://www2.darwin.edu.ar. (Fecha de consulta junio de 2020). 